\title{
Orphan Drugs in Development for the Treatment of Small-Cell Lung Cancer: Emerging Data on Lurbinectedin
}

\author{
Diego Kauffmann-Guerrero (ID) \\ Rudolf Maria Huber (iD \\ Division of Respiratory Medicine and \\ Thoracic Oncology, Department of \\ Internal Medicine $V$ and Thoracic \\ Oncology Centre Munich, University of \\ Munich (LMU), Comprehensive \\ Pneumology Center, Member of the \\ German Center for Lung Research \\ (DZL), Munich, Germany
}

This article was published in the following Dove Press journal: Lung Cancer: Targets and Therapy

\begin{abstract}
Lung cancer is the leading cause of death of all cancer entities and small-cell lung cancer (SCLC) is the most malignant subtype. Despite good initial response to chemotherapy, many patients relapse early and success of second line treatment remains poor. For years, no relevant improvement of second line treatment has been achieved in the field of SCLC. Lurbinectedin, a novel RNA-polymerase II inhibitor has shown promising results in pretreated SCLC patients as single agent and in combination with other chemotherapeutic drugs leading to an orphan drug designation from the FDA. This article reviews the current data on this emerging substance and its impact on the treatment of SCLC.
\end{abstract}

Keywords: SCLC, chemotherapy, lurbinectedin, orphan drug

\section{Introduction}

Small-cell lung cancer (SCLC) accounts for about $15 \%$ to $17 \%$ of all diagnosed lung cancers. Nevertheless, due to its aggressive and rapid behavior, SCLC is the leading cause of death among all malignancies. ${ }^{1}$ Promising progress in the field of non-small cell cancer (NSCLC) regarding targeted therapy and immunotherapy has been achieved in the last few years. However, the prognosis and therapeutic options of SCLC are still limited with a median survival of patients with extensive disease between 7 to 10 months and a 1-year survival of $20 \%$ to $40 \%{ }^{2}$

Despite the slight improvement of overall survival by adding checkpoint inhibitors to first line treatment, ${ }^{3}$ and the development of other various treatment options, eg, PARP inhibitors, or cell cycle modulating agents, chemotherapy remains the backbone of SCLC therapy. ${ }^{4}$

Despite the good response of first line chemotherapy in SCLC, patients generally relapse early and therapeutic options in second line treatment are limited. In sensitive disease, topotecan, which is the current standard second line treatment in Europe, irinotecan and amrubicin have shown modest activity as monotherapy, ${ }^{5-7}$ while doxorubicin and ifosfamide were revealed not to be effective in refractory relapse. ${ }^{8,9}$ Given the disappointing results in second line therapy, new therapeutic approaches are desperately needed in the field of SCLC.

Lurbinectedin is a novel RNA-polymerase-II inhibitor showing promising results in several cancer entities. Also in SCLC, lurbinectedin has proven relevant activity leading to an orphan drug designation from the FDA in August 2018.
Correspondence: Diego KauffmannGuerrero

Hospital of the University of Munich (LMU), Ziemssenstraße I, Munich 80336 Germany

Tel +49-89-4400-52187

Email KauffmannGuerrero@med.unimuenchen.de 
In this article, we review the current literature of preclinical and clinical data on lurbinectedin in SCLC treatment.

\section{Mechanism of Action and Preclinical Data of Lurbinectedin}

Lurbinectedin (PM01183) is a derivative of ecteinascidin, a marine-derived agent that covalently binds to the DNA minor groove and thus leads to double-strand DNA breaks. Furthermore, it inhibits RNA-polymerase-II activity and promotes its specific degradation by the ubiquitin/proteasome machinery. ${ }^{10,11}$ Lurbinectedin is a second-generation trabectedin analog with similar structure except for the C subunit, where tetrahydroisoquinoline was replaced by a tetrahydro $\beta$-carboline in lurbinectedin. ${ }^{10,12}$ This difference may have an impact on pharmacokinetics and pharmacodynamics. It has been proposed that modification of the C-ring could enhance the direct interactions with specific factors of DNA repair. ${ }^{10,13,14}$ It has been shown to have potent cytotoxic activity in several cell lines and murine xenograft human cancer models. ${ }^{10}$ Furthermore, by attenuating the activity of the nucleotide excision repair (NER) mechanism, lurbinectedin was able to overcome cisplatin resistance in NER hyperactive cell lines. ${ }^{12}$ Single lurbinectedin as well as in combination with cisplatin was effective in cisplatin-resistant ovarian tumor models. ${ }^{15,16}$ Also, cervical cell lines and cervical cancers in xenograft mouse models were highly affected by single agent lurbinectedin. ${ }^{17}$ Lurbinectedin was also shown to inactivate Ewing Sarcoma Oncoprotein (EWS-FLI1) by nuclear redistribution leading to promotor inactivity and decreased mRNA and protein levels. ${ }^{18}$

\section{Clinical Development of Lurbinectedin}

In 2014, Elez et al reported the first-in-human results of a Phase I dose finding study. They treated 31 patients with solid tumors and increasing doses of lurbinectedin. $7.0 \mathrm{mg}$ as flat dose was recommended as a $1 \mathrm{hr}$ infusion q3wk. ${ }^{19}$ Neutropenia and febrile infections were the dose limiting adverse events. ${ }^{19}$ Because of severe hematological side effects a second Phase I dose finding study was performed and recommended a flat dose of $5 \mathrm{mg}$ of lurbinectedin given as $1 \mathrm{hr}$ infusion on day 1 and 8 every 3 weeks. ${ }^{20}$ A further Phase I study evaluated the recommended dose for the combination of lurbinectedin and gemcitabine
(3 $\mathrm{mg}$ lurbinectedin and $800 \mathrm{mg} / \mathrm{m} 2$ gemcitabine given on day 1 and 8 every 3 weeks). ${ }^{21}$

A case study of two patients with mesothelioma reported disease stabilization in both patients for 5.5 and 6 months in second line treatment combining cisplatin and lurbinectedin. $^{22}$

After promising preclinical results in ovarian cancer, lurbinectedin was tested in a randomized Phase II study versus topotecan in patients with platinum refractory ovarian cancer and showed a $23 \%$ response rate. ${ }^{23}$ There are also hints of some activity of lurbinectedin in BRCA mutated breast cancers. ${ }^{24}$

Despite good response in gynecological tumors, a Phase I study in patients with acute myeloid leukemia and myelodysplastic syndrome did not show a sustainable effect. ${ }^{25}$

\section{Lurbinectedin in SCLC}

As a promising chemotherapeutic agent in several tumor types, lurbinectedin was also evaluated in SCLC. Furthermore, RNA-polymerase II is commonly hyperactivated in SCLC indicating a good point of attack. ${ }^{26}$

A Phase I study combining doxorubicin and lurbinectedin in 27 relapsed SCLC patients found remarkable activity in a second line setting. ${ }^{27} 91.7 \%$ of patients with platinum-sensitive disease and $33.3 \%$ of patients with resistant disease did respond to the combinational therapy. The progression free survival (PFS) was 5.8 and 3.5 months respectively. In third line, $20 \%$ of patients, all with resistant disease, responded to doxorubicin and lurbinectedin with a median PFS of 1.2 months. ${ }^{27}$ An expansion cohort with reduced dose was implemented in this study to improve safety. Patients with no more than one prior chemotherapy line and stable brain metastases were included. Doxorubicin was interrupted after 10 cycles continuing with lurbinectedin alone.

Overall confirmed ORR was $37 \%$ in resistant patients and $53 \%$ in patients with sensitive disease. Overall median PFS was 3.4 months (95\% CI, 1.5-6.2), being 1.5 months (95\% CI, 0.8-3.4) in resistant patients, and 5.7 months in sensitive patients. Overall median OS was 7.9 months (95\% CI, 4.9-11.5), 4.9 months (95\% CI, 2.3-6.7) in resistant and 11.5 months $(95 \% \mathrm{CI}, 6.0-16.6)$ in sensitive patients. ${ }^{28}$

Recently, the results of a Phase II basket trial investigating the safety and efficacy of lurbinectedin as a single agent in several tumor types were presented. 105 patients with SCLC were enrolled in the study. They had to have at least one prior chemotherapy session. Patients with CNS metastases were excluded from the study. The overall 


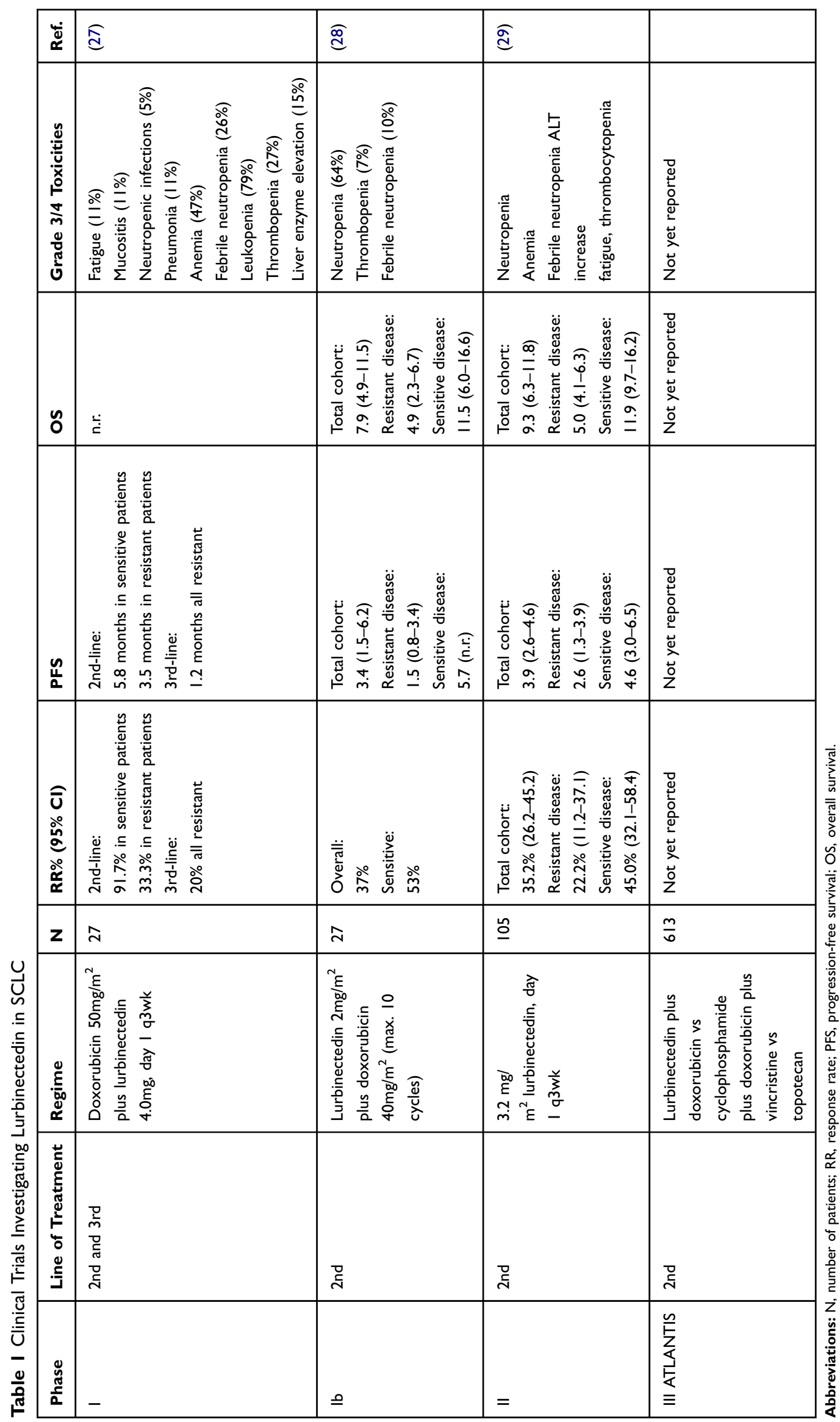


response rate was $35.2 \%$ (95\% CI, 26.2-45.2). Median PFS was 5.3 months (95\% CI, 3.5-6.4) and median OS 10.8 months (95\% CI, 6.5-12.2). ${ }^{29}$ Patients with sensitive disease had a better response and outcome than patients with resistant disease.

For comparison, the pivotal study of topotecan, the current standard in second line treatment in relapsed disease, revealed an ORR of $24.3 \%$, a median PFS of 13.3 weeks, and a median OS of 25.0 weeks. ${ }^{5}$ Besides, irinotecan as monotherapy in pretreated patients showed an ORR of $17.5 \%$, a median PFS of 11.3 weeks, and a median OS of 13.3 weeks. $^{6}$ The topoisomerase II inhibitor amrubicin has also been investigated in second-line treatment. Kimura et al performed a meta-analysis of 296 SCLC patients treated with amrubicin. Even patients with refractory disease achieved an ORR of $36.8 \%$ and survival of 5.3 to 11 months. $^{7}$

The ongoing ATLANTIS trial (NCT02566993) is a Phase III study investigating the combination of lurbinectedin and doxorubicin compared with either cyclophosphamide, doxorubicin, and vincristine (CAV) or topotecan in patients with relapsed SCLC, with a primary endpoint of OS. The trial has completed enrollment and results are expected next year. Table 1 summarizes the clinical trials investigating lurbinectedin in SCLC. Severe adverse events were relatively frequent in all lurbinectedin studies. Especially hematological side effects such as neutropenia and thrombopenia were typical events. Table 1 summarizes the grade 3 and 4 toxicities. However, the frequency of severe adverse events in patients with lurbinectedin alone is comparable with that seen in topotecan treatment.

\section{Summary and Outlook}

In this article, we reviewed the current data on lurbinectedin in the treatment of SCLC. The results of the Phase I and Phase II trials proved lurbinectedin to be a promising agent with potent antitumor activity. Especially in patients with platinum-sensitive disease, the activity of lurbinectedin seems to be notably high. This could be a subgroup of patients that in particular might benefit from lurbinectedin in second line treatment. Nevertheless, in SCLC many promising results from Phase I/II studies turned out to be negative in randomized trials. That is why the results of the randomized ATLANTIS trial are wishfully awaited. If they can confirm the earlier study results lurbinectedin will probably become a new standard in second line treatment of SCLC.
However, taking into account the increasing impact of immunotherapy, also in the field of SCLC, every novel agent will be faced with new standards of care in first and second line. Accordingly, new randomized studies are needed to evaluate the significance of lurbinectedin as single agent or in combination with chemotherapy and/or checkpoint inhibitors in the treatment of SCLC patients.

\section{Disclosure}

Prof. Dr. Rudolf M Huber reports personal fees from AstraZeneca Germany, personal fees from BMS Germany, personal fees from Bayer, personal fees from Boehringer Ingelheim Germany, personal fees from Lilly Germany, personal fees from MSD Germany, personal fees from Novartis Germany, personal fees from Pfizer Germany, personal fees from Roche Germany, and personal fees from Takeda, outside the submitted work. The authors report no other conflicts of interest in this work.

\section{References}

1. Torre LA, Bray F, Siegel RL, Ferlay J, Lortet-Tieulent J, Jemal A. Global cancer statistics, 2012. CA Cancer J Clin. 2015;65(2):87-108. doi: $10.3322 /$ caac. 21262

2. Kahnert K, Kauffmann-Guerrero D, Huber RM. SCLC-state of the art and what does the future have in store? Clin Lung Cancer. 2016;17 (5):325-333. doi:10.1016/j.cllc.2016.05.014

3. Horn L, Mansfield AS, Szczesna A, et al. First-line atezolizumab plus chemotherapy in extensive-stage small-cell lung cancer. $N$ Engl J Med. 2018;379(23):2220-2229. doi:10.1056/NEJMoa1809064

4. Zimmerman S, Das A, Wang S, Julian R, Gandhi L, Wolf J. 2017-2018 Scientific advances in thoracic oncology: small cell lung cancer. $J$ Thorac Oncol. 2019;14(5):768-783. doi:10.1016/j.jtho.20 19.01.022

5. von Pawel J, Schiller JH, Shepherd FA, et al. Topotecan versus cyclophosphamide, doxorubicin, and vincristine for the treatment of recurrent small-cell lung cancer. J Clin Oncol. 1999;17(2):658-667. doi:10.1200/JCO.1999.17.2.658

6. Sevinc A, Kalender ME, Altinbas $M$, et al. Irinotecan as a second-line monotherapy for small cell lung cancer. Asian Pac J Cancer Prev. 2011;12(4):1055-1059.

7. Kimura T, Kudoh S, Hirata K. Review of the management of relapsed small-cell lung cancer with amrubicin hydrochloride. Clin Med Insights Oncol. 2011;5:23-34. doi:10.4137/CMO.S5072

8. Tanaka I, Kawada K, Morise M, et al. A phase II trial of Ifosfamide combination with recommended supportive therapy for recurrent SCLC in second-line and heavily treated setting. Cancer Chemother Pharmacol. 2018;81(2):339-345. doi:10.1007/ s00280-017-3497-0

9. Samantas E, Kalofonos H, Linardou H, et al. Phase II study of pegylated liposomal doxorubicin: inactive in recurrent small-cell lung cancer. A hellenic cooperative oncology group study. Ann Oncol. 2000;11(11):1395-1397. doi:10.1023/A:1026523316736

10. Leal JF, Martinez-Diez M, Garcia-Hernandez V, et al. PM01183, a new DNA minor groove covalent binder with potent in vitro and in vivo anti-tumour activity. $B r J$ Pharmacol. 2010;161(5):10 99-1110. doi:10.1111/j.1476-5381.2010.00945.x 
11. Santamaria Nunez G, Robles CM, Giraudon C, et al. Lurbinectedin specifically triggers the degradation of phosphorylated RNA polymerase II and the formation of DNA breaks in cancer cells. Mol Cancer Ther. 2016;15(10):2399-2412. doi:10.1158/1535-7163.MCT16-0172

12. Soares DG, Machado MS, Rocca CJ, et al. Trabectedin and its C subunit modified analogue PM01183 attenuate nucleotide excision repair and show activity toward platinum-resistant cells. Mol Cancer Ther. 2011;10(8):1481-1489. doi:10.1158/1535-7163. MCT-11-0252

13. Casado JA, Rio P, Marco E, et al. Relevance of the Fanconi anemia pathway in the response of human cells to trabectedin. Mol Cancer Ther. 2008;7(5):1309-1318. doi:10.1158/1535-7163. MCT-07-2432

14. Herrero AB, Martin-Castellanos C, Marco E, Gago F, Moreno S. Cross-talk between nucleotide excision and homologous recombination DNA repair pathways in the mechanism of action of antitumor trabectedin. Cancer Res. 2006;66(16):8155-8162. doi:10.1158/00085472.CAN-06-0179

15. Vidal A, Munoz C, Guillen MJ, et al. Lurbinectedin (PM01183), a new DNA minor groove binder, inhibits growth of orthotopic primary graft of cisplatin-resistant epithelial ovarian cancer. Clin Cancer Res. 2012;18 (19):5399-5411. doi:10.1158/1078-0432.CCR-12-1513

16. Takahashi R, Mabuchi S, Kawano M, et al. Preclinical Investigations of PM01183 (Lurbinectedin) as a single agent or in combination with other anticancer agents for clear cell carcinoma of the ovary. PLoS One. 2016;11(3):e0151050. doi:10.1371/ journal.pone.0151050

17. Yokoi E, Mabuchi S, Shimura K, et al. Lurbinectedin (PM01183), a selective inhibitor of active transcription, effectively eliminates both cancer cells and cancer stem cells in preclinical models of uterine cervical cancer. Invest New Drugs. 2018;37(5):818-827. doi:10.1007/s10637-018-0686-6

18. Harlow ML, Maloney N, Roland J, et al. Lurbinectedin inactivates the ewing sarcoma oncoprotein EWS-FLI1 by redistributing it within the nucleus. Cancer Res. 2016;76(22):6657-6668. doi:10.1158/00085472.CAN-16-0568

19. Elez ME, Tabernero J, Geary D, et al. First-in-human phase I study of Lurbinectedin (PM01183) in patients with advanced solid tumors. Clin Cancer Res. 2014;20(8):2205-2214. doi:10.1158/1078-0432.CCR-131880
20. Jimeno A, Sharma MR, Szyldergemajn S, et al. Phase I study of lurbinectedin, a synthetic tetrahydroisoquinoline that inhibits activated transcription, induces DNA single- and double-strand breaks, on a weekly x 2 every-3-week schedule. Invest New Drugs. 2017;35 (4):471-477. doi:10.1007/s10637-017-0427-2

21. Paz-Ares L, Forster M, Boni V, et al. Phase I clinical and pharmacokinetic study of PM01183 (a tetrahydroisoquinoline, Lurbinectedin) in combination with gemcitabine in patients with advanced solid tumors. Invest New Drugs. 2017;35(2):198-206. doi:10.1007/s106 37-016-0410-3

22. Metaxas Y, Cathomas R, Mark M, von Moos R. Combination of cisplatin and lurbinectedin as palliative chemotherapy in progressive malignant pleural mesothelioma: report of two cases. Lung Cancer. 2016;102:136-138. doi:10.1016/j.lungcan.2016.07.012

23. Poveda A, Del Campo JM, Ray-Coquard I, et al. Phase II randomized study of PM01183 versus topotecan in patients with platinum-resistant/ refractory advanced ovarian cancer. Ann Oncol. 2017;28(6):12 80-1287. doi:10.1093/annonc/mdx111

24. Gourd E. Lurbinectedin for BRCA-mutated advanced breast cancer. Lancet Oncol. 2018;19(11):e582. doi:10.1016/S1470-2045(18)307 $37-\mathrm{X}$

25. Benton CB, Chien KS, Tefferi A, et al. Safety and tolerability of lurbinectedin (PM01183) in patients with acute myeloid leukemia and myelodysplastic syndrome. Hematol Oncol. 2019;37(1):96-102. doi:10.1002/hon.v37.1

26. Fernandez-Teruel C, Gonzalez I, Troconiz IF, Lubomirov R, Soto A, Fudio S. Population-Pharmacokinetic and covariate analysis of lurbinectedin (PM01183), a new RNA polymerase II inhibitor, in pooled Phase I/II trials in patients with cancer. Clin Pharmacokinet. 2019;58 (3):363-374. doi:10.1007/s40262-018-0701-2

27. Calvo E, Moreno V, Flynn M, et al. Antitumor activity of lurbinectedin (PM01183) and doxorubicin in relapsed small-cell lung cancer: results from a phase I study. Ann Oncol. 2017;28(10):2559-2566. doi:10.1093/annonc/mdx357

28. Forster M, Moreno V, Calvo E, et al. P1.12-20 Overall survival with lurbinectedin plus doxorubicin in relapsed SCLC. Results from an expansion Cohort of a Phase Ib trial. J Thorac Oncol. 2018;13(10): S581. doi:10.1016/j.jtho.2018.08.855

29. Paz-Ares LG, Perez JMT, Besse B, et al. Efficacy and safety profile of lurbinectedin in second-line SCLC patients: results from a phase II single-agent trial. J Clin Oncol. 2019;37(15_suppl):8506.
Lung Cancer: Targets and Therapy

\section{Publish your work in this journal}

Lung Cancer: Targets and Therapy is an international, peerreviewed, open access journal focusing on lung cancer research, identification of therapeutic targets and the optimal use of preventative and integrated treatment interventions to achieve improved outcomes, enhanced survival and quality of life for the cancer patient. Specific topics covered in the journal include: Epidemiology, detection and screening; Cellular research and biomarkers; Identification of biotargets and agents with novel mechanisms of action; Optimal clinical use of existing anticancer agents, including combination therapies; Radiation and surgery; Palliative care; Patient adherence, quality of life, satisfaction; Health economic evaluations. 